Journal of Organometallic Chemistry, 367 (1989) 197-204

Elsevier Sequoia S.A., Lausanne - Printed in The Netherlands

JOM 09720

\title{
Reaktivität der Metall-Metall-Mehrfachbindung in Übergangsmetall-Komplexen
}

\section{XIV *. Reaktion von $\mathrm{Cp}_{2}^{\star} \mathrm{CoRh}(\mu-\mathrm{CO})_{2}\left(\mathrm{Cp}^{\star}=\eta^{5}-\mathrm{C}_{5} \mathrm{Me}_{5}\right)$ \\ mit Schwefel oder Selen und Molekülstruktur von $\mathrm{Cp}_{2}^{\star} \mathrm{CoRh}(\mathrm{CO})_{2}(\mu-\mathrm{Se})_{2}$}

\author{
Henri Brunner, Norbert Janietz, Joachim Wachter * \\ Institut für Anorganische Chemie der Universitüt Regensburg, Universitätsstr. 31, \\ D-8400 Regensburg (B.R.D.)
}

Bernd Nuber und Manfred L. Ziegler

Anorganisch-Chemisches Institut der Universität Heidelberg, Im Neuenheimer Feld 270, D.6900 Heidelberg (B.R.D.)

(Eingegangen den 15. Dezember 1988)

\begin{abstract}
The reaction of $\mathrm{Cp}_{2}^{\star} \mathrm{CoRh}(\mu-\mathrm{CO})_{2}\left(M=M ; \mathrm{Cp}^{\star}=\eta^{5}-\mathrm{C}_{5} \mathrm{Me}_{5}\right)$ with elemental sulfur or selenium gives the complexes $\mathrm{Cp}_{2}^{\star} \mathrm{CoRhS}$ and $\mathrm{Cp}_{2}^{\star} \mathrm{CoRhSe} \mathrm{s}_{5}$, both are distinguished by a high chalcogen content. The only $\mathrm{CO}$ containing intermediate product $\mathrm{Cp}_{2}^{\star} \mathrm{CoRh}(\mathrm{CO})_{2} \mathrm{Se}_{2}$ that was isolated has been characterized by an X-ray diffraction study. The compound contains a rhombohedric, planar $M_{2} E_{2}$ four-membered ring (angle $\mathrm{Co}-\mathrm{Se}-\mathrm{Rh} 101.3(1)^{\circ}$ ) bonded to anti-oriented $\mathrm{Cp}^{\star}$ and $\mathrm{CO}$ ligands. Other $\mathrm{CO}$-containing intermediates can be detected only spectroscopically. Thus the Co atom in the $\mathbf{M}-\mathbf{M}$ double bond exerts an accelerating influence on the reaction rate when compared to $R h$.
\end{abstract}

\section{Zusammenfassung}

Die Reaktion von $\left.\mathrm{Cp}_{2}^{\star} \mathrm{CoRh}(\mu-\mathrm{CO})_{2}\right)\left(M=M ; \mathrm{Cp}^{\star}=\eta^{5}-\mathrm{C}_{5} \mathrm{Me}_{5}\right)$ mit elementarem Schwefel oder Selen führt zu den chalkogenreichen Komplexen $\mathrm{Cp}_{2}^{\star} \mathrm{CoRhS}_{8}$ und $\mathrm{Cp}_{2}^{\star} \mathrm{CoRhSe}_{5}$. Als einziges CO-haltiges Zwischenprodukt konnte $\mathrm{Cp}_{2}^{\star} \mathrm{CoRh}(\mathrm{CO})_{2} \mathrm{Se}_{2}$ in Substanz isoliert und durch eine Röntgenstrukturanalyse charakterisiert werden.

* XIII. Mitteilung siehe Ref. 1. 
Die Verbindung enthält einen rhomboedrischen, planaren $\mathrm{M}_{2} \mathrm{E}_{2}$-Vierring (Winkel $\mathrm{Co}-\mathrm{Se}-\mathrm{Rh} 101.3(1)^{\circ}$ ) mit dazu anti-ständigen $\mathrm{Cp}^{\star}$ - und CO-Liganden. Weitere CO-haltige Zwischenstufen können nur spektroskopisch erfaßt werden. Hieraus kann auf einen reaktionsbeschleunigenden Einfluß des Co-Atoms in der $\mathbf{M}-\mathbf{M}$ Doppelbindung im Vergleich zu Rh geschlossen werden.

\section{Einleitung}

Die M-M-Doppelbindungen der Komplexe $\mathrm{Cp}_{2}^{\star} \mathbf{M}_{2}(\mu-\mathrm{CO})_{2}\left(\mathbf{M}=\mathrm{Co}, \mathbf{R h} ; \mathrm{Cp}^{\star}\right.$ $\left.=\eta^{5}-\mathrm{C}_{5} \mathrm{Me}_{5}\right)$ sind in der Lage, ein oder zwei Äquivalente an elementarem Schwefel oder Selen zu addieren [1]. Bei Verwendung von überschüssigem Chalkogen erhält man dagegen CO-freie, diamagnetische Verbindungen von erheblich höherem Chalkogengehalt [1,2]. Da sowohl die Konstitution als auch die Stabilität der gebildeten Produkte nicht nur vom eingesetzten Chalkogen, sondern auch von der Natur des Übergangsmetalls bestimmt werden, lag es nunmehr nahe, die Reaktion des gemischtkernigen Komplexes $\mathrm{Cp}_{2}^{\star} \mathrm{CoRh}(\mu-\mathrm{CO})_{2}$ (I) mit den entsprechenden Chalkogenen $\mathrm{zu}$ untersuchen. Unterschiede in der Reaktivität von $3 d$ - und $4 d$ Übergangsmetallmehrfachbindungssystemen gegenüber Chalkogenen sind außer für die Elemente $\mathrm{Co}$ und $\mathrm{Rh}$ bisher nur für die Elemente $\mathrm{Cr}$, Mo und $\mathrm{W}$ untersucht worden [2,3]. Untersuchungen über gemischtkernige Komplexe liegen dagegen nicht vor.

\section{Die Reaktion von $\mathrm{Cp}_{2}^{\star} \mathrm{CoRh}(\mathrm{CO})_{2}$ mit Schwefel}

Setzt man die blaugrüne THF-Lösung von I mit einem Moläquivalent $S_{8}$ bei Raumtemperatur um, so bildet sich unter rascher Farbänderung ein CO-freier, rotbrauner Komplex II (Gl. 1). Analytische und massenspektroskopische [4] Untersuchungen belegen, daß es sich hierbei um einen schwefelreichen Komplex der Zusammensetzung $\mathrm{Cp}_{2}^{\star} \mathrm{CoRhS}_{8}$ handelt, der analog zu dem bereits röntgenstrukturanalytisch gesicherten $\mathrm{Cp}_{2}^{\star} \mathrm{Rh}_{2} \mathrm{~S}_{8}$ [5] aufgebaut sein könnte. Komplex II wäre demnach durch je einen $\mathrm{CoS}_{4}$ - und einen $\mathrm{RhS}_{4}$-Chelatring charakterisiert. Bedingt durch die asymmetrische Verknüpfung zu einem $\mathrm{CoRhS}_{3}$-Fünfring wären die zwei in Gl. 1 aufgeführten Strukturisomere möglich. Im ${ }^{1} \mathrm{H}-\mathrm{NMR}$-Spektrum lassen sich jedoch anstelle der erwarteten vier Signale für die $\mathrm{CH}_{3}$-Protonen nur zwei Singuletts, nämlich bei 1.63 und $1.67 \mathrm{ppm}$ beobachten, was auf die Existenz nur eines Isomeren hindeutet und eventuell auf einen steuernden Einfluß des Co-Atoms zurückzuführen ist. In diesem Zusammenhang muß vermerkt werden, daß die Präsenz zweier Co-Atome in M-M-Doppelbindungssystem zur Bildung nur eines einzigen Produkts $\left(\mathrm{Cp}_{2}^{\star} \mathrm{Co}_{2} \mathrm{~S}_{4}, \mathrm{VI}\right)$ führt [6].

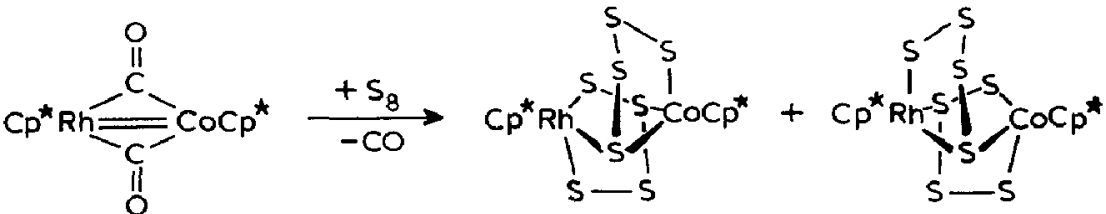




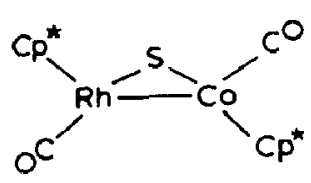

(III)<smiles>[O][Pb]1([O-])SC([18O])([Se])S1</smiles>

(IV)

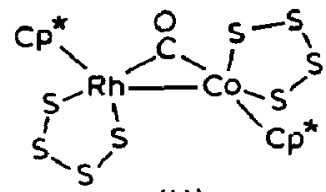

(v)

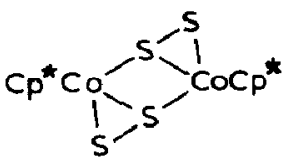

(VI)

Schema 1

Vergleicht man die Reaktivität der Co=Rh-Bindung in I mit derjenigen des analogen $R h=R h-K o m p l e x e s$, so findet man eine drastische Beschleunigung der Reaktionsgeschwindigkeit durch Co: Die Addukte III-V (Schema 1) bilden sich bereits bei $-60^{\circ} \mathrm{C}$, was sich IR-spektroskopisch beweisen läßt ( $\nu(\mathrm{CO})$ : III: 1960; IV: $2020 ; \mathrm{V}: 1812 \mathrm{~cm}^{-1}$ ). Im Gegensatz zu den analogen Dirhodiumkomplexen disproportionieren ihre Lösungen entweder bereits bei tiefen Temperaturen oder reagieren zu Il weiter.

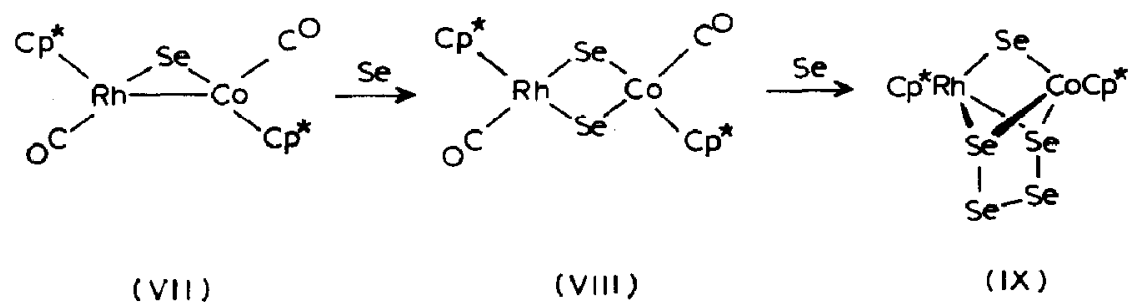

Die Reaktion von $\mathrm{Cp}_{2}^{\star} \mathrm{CoRh}(\mathrm{CO})_{2}$ mit Selen

Die Addition von Selen an die $\mathrm{C} \sigma=\mathrm{Rh}$-Bindung von I verläuft stufenweise (Gl. 2). Allerdings ist das zuerst gebildete Monoaddukt VII nur IR-spektroskopisch nachzuweisen ( $\left.\nu(C O) 1955 \mathrm{~cm}^{-1}\right)$, es disproportioniert in Lösung rasch zu I und dem Diaddukt VIII. Damit ist VII bezüglich seiner Stabilität in etwa zwischen seine beiden homonuklearen Homologen einzuordnen: während $\mathrm{Cp}_{2}^{\star} \mathrm{Co}_{2}(\mathrm{CO})_{2}(\mu$-Se) bisher noch nicht nachgewiesen werden konnte, gelang die Synthese von $\mathrm{Cp}_{2}^{\star} \mathrm{Rh}_{2}(\mathrm{CO})_{2}(\mu-\mathrm{Se})$ problemlos [1,7]. Strukturell ist dieser Komplex durch einen $\mathrm{Rh}_{2}$ Se-Dreiring charakterisiert, in dem Se als 2-e-Ligand fungiert [8]. Die Beteiligung von $\mathrm{Co}$ in diesem Bindungssystem könnte jedoch bedeuten, daß die M-M-Bindung zugunsten von M-Chalkogendoppelbindungen aufgegeben wird, womit die Brückenliganden formal zu 4-e-Liganden umgewandelt würden. Dieser unterschiedliche Sachverhalt für strukturell analoge $3 d$-bzw. $4 d$-Komplexe konnte am Beispiel der Verbindungsklasse $\left[\mathrm{Cp}^{\star} \mathrm{M}(\mathrm{CO})_{2}\right]_{2}(\mu-\mathrm{S})(\mathrm{M}=\mathrm{Mn}, \mathrm{Re})$ stichhaltig belegt werden [9].

Der grünblaue Komplex VIII läßt sich gezielt durch Umsetzung von drei Äquivalenten gealtertem roten Selen (frisch hergestelltes rotes Selen ist zu reaktiv 
Tabelle 1

Spektroskopische Daten der Komplexe II-V, VII-IX

\begin{tabular}{lccl}
\hline & IR $\left(\mathrm{cm}^{-1}, \mathrm{KBr}\right)$ & $\begin{array}{l}{ }^{1} \mathrm{H}_{-} \mathrm{NMR}^{\mathrm{c}} \\
\left(\delta\left(\mathrm{CH}_{3}\right)\right)\end{array}$ \\
\cline { 2 - 3 } & $\boldsymbol{\nu}(\mathrm{CO})$ & $\nu(\mathrm{M}-\mathrm{CO})$ & $1.63,1.67$ \\
II & - & & $a$ \\
III & $1960^{a}$ & & $a$ \\
IV & $2020^{a}$ & & $a$ \\
V & $1812^{a}$ & & $1.82,1.91$ \\
VII & $1955^{b}$ & $500 \mathrm{~s}, 490 \mathrm{~m}, 480 \mathrm{~m}$ & $1.57,1.61$ \\
VIII & $1990 \mathrm{vs}$ & & \\
IX & - & & \\
\hline
\end{tabular}

${ }^{a}$ Siehe Text. ${ }^{b}$ THF-Lösung. ${ }^{c} \mathrm{CDCl}_{3}$-Lösung, i-TMS; Varian EM-360L Spektrometer $\left(33^{\circ} \mathrm{C}\right)$.

und führt sofort zu Totalsubstitution von $\mathrm{CO}$ ) mit I bei Raumtemperatur darstellen. Im FD-Massenspektrum läßt sich die sukzessive Abspaltung der CO-Gruppen vom Molekülpeak verfolgen. Die massenspektroskopisch festgestellte Labilität der terminalen CO-Liganden $\left(\nu(C O) 1990 \mathrm{~cm}^{-1}\right)$ ist auch präparativ nachvollziehbar. Beim Erwärmen einer Toluollösung von I bildet sich ein Komplex der Zusammensetzung $\mathrm{Cp}_{4}^{\star} \mathrm{Co}_{2} \mathrm{Rh}_{2} \mathrm{Se}_{4}$, dessen Struktur Gegenstand derzeitiger Untersuchungen ist.

Der CO-freie Komplex IX ist aus I mit überschüssigem Selen darstellbar. Wie bereits in $\mathrm{Cp}_{2}^{\star} \mathrm{Co}_{2} \mathrm{Se}_{5}$ [1] und $\mathrm{Cp}_{2}^{\star} \mathrm{Rh}_{2} \mathrm{Se}_{5}$ [10] sollten die beiden Metallatome durch einen $\mathrm{Se}_{4}{ }^{2-}$ - und einen $\mathrm{Se}^{2-}$-Liganden verbrückt sein unter Ausbildung eines nahezu planaren Pseudofünfrings, der die Co-Rh-Achse halbiert und auf dieser senkrecht steht. Trotz der symmetrischen Anordnung der Liganden werden im ${ }^{1}$ H-NMRSpektrum zwei Resonanzen für die $\mathrm{CH}_{3}$-Protonen gefunden (Tab. 1), was wie in VIII auf die unterschiedliche Natur der Metallatome zurückzuführen ist.

Tabelle 2

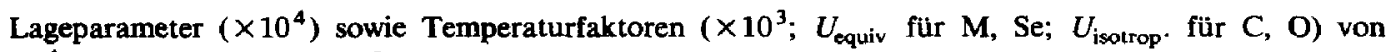
$\mathrm{Cp}_{2}^{\star} \mathrm{CoRh}(\mathrm{CO})_{2} \mathrm{Se}_{2}$ (VIII) ${ }^{a}$

\begin{tabular}{lrrrr}
\hline Atom & \multicolumn{1}{l}{$y$} & \multicolumn{1}{c}{$z$} & \multicolumn{1}{c}{$U^{a}$} \\
\hline M & $925(2)$ & $273(2)$ & $9048(1)$ & $35(1)$ \\
Se(1) & $169(2)$ & $154(3)$ & $10640(1)$ & $50(1)$ \\
C(11) & $1234(37)$ & $-1587(19)$ & $9020(18)$ & $116(16)$ \\
O(11) & $1475(25)$ & $-2723(15)$ & $8985(14)$ & $131(11)$ \\
C(1) & $2426(22)$ & $998(18)$ & $8186(11)$ & $41(7)$ \\
C(2) & $816(22)$ & $1028(18)$ & $7738(11)$ & $36(7)$ \\
C(3) & $-52(25)$ & $2029(23)$ & $8106(16)$ & $54(9)$ \\
C(4) & $1176(35)$ & $2531(18)$ & $8872(17)$ & $59(11)$ \\
C(5) & $2766(24)$ & $1875(18)$ & $8984(12)$ & $47(8)$ \\
C(6) & $3844(22)$ & $118(22)$ & $7959(14)$ & $78(10)$ \\
$C(7)$ & $68(26)$ & $285(28)$ & $6853(14)$ & $106(13)$ \\
$C(8)$ & $-1732(23)$ & $2462(32)$ & $7747(20)$ & $131(16)$ \\
C(9) & $931(43)$ & $3668(21)$ & $9539(22)$ & $170(22)$ \\
C(10) & $4297(25)$ & $2122(23)$ & $9628(14)$ & $71(11)$ \\
\hline
\end{tabular}

a $U_{\text {equiv }}=1 / 3$ Spur $U ; \mathrm{M}$ steht für $\mathrm{Rh}$ und $\mathrm{Co}(1 / 1$ statistisch verteilt). 
Tabelle 3

Ausgewählte Bindungslängen ( $\AA$ ) und -winkel ( $\left.{ }^{\circ}\right)$ für $\mathrm{Cp}_{2}^{\star} \mathrm{CoRh}(\mathrm{CO})_{2} \mathrm{Se}_{2}$ (VIII) ${ }^{a}$

\begin{tabular}{lllc}
\hline$M-S e(1)$ & $2.406(2)$ & $\operatorname{Se}(1)-M-S e\left(1^{\prime}\right)$ & $78.7(1)$ \\
$M-S e\left(1^{\prime}\right)$ & $2.411(3)$ & $\operatorname{Se}(1)-M-C(11)$ & $88.7(9)$ \\
$M-C(1)$ & $2.18(2)$ & $\operatorname{Se}\left(1^{\prime}\right)-M-C(11)$ & $89.1(1.1)$ \\
$M-C(2)$ & $2.15(2)$ & $M-S e(1)-M^{\prime}$ & $101.3(1)$ \\
$M-C(3)$ & $2.24(2)$ & & \\
$M-C(4)$ & $2.18(2)$ & & \\
$M-C(5)$ & $2.20(2)$ & & \\
$M-C(11)$ & $1.80(2)$ & & \\
Se(1) $\cdots\left(\operatorname{Se}\left(1^{\prime}\right)\right.$ & $3.055(3)$ & & \\
\hline
\end{tabular}

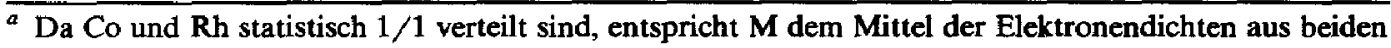
Elementen.

Die Molekiulstruktur von $\mathrm{Cp}_{2}^{\star} \mathrm{CoRh}(\mathrm{CO})_{2} \mathrm{Se}_{2}(\mathrm{VIII})$

Komplexe des Typs $\mathrm{Cp}_{2}^{\star} \mathrm{M}_{2}(\mathrm{CO})_{2} \mathrm{E}_{2}(\mathrm{M}=\mathrm{Co}, \mathrm{Rh} ; \mathrm{E}=\mathrm{S}$, Se, Te) können prinzipiell entweder zwei $\mathrm{Se}^{2-}$ - oder eine $\eta^{1}-\mathrm{Se}_{2}{ }^{2-}$-Brücke enthalten, die zwar beide je acht Elektronen zur Gesamtelektronenbilanz beitragen (letztere u. a. über $p_{\pi^{-}}-d_{\pi^{-}}$ Wechselwirkungen), jedoch unterschiedliche Formalladungen der Zentralmetallatome zur Folge haben. Während der zweite Ligandentyp bereits in Komplexen vom Typ $\left[\mathrm{C}_{5} \mathrm{R}_{5} \mathrm{M}(\mathrm{CO})_{2}\right] \mathrm{E}_{2}(\mathrm{M}=\mathrm{Mn}, \mathrm{Re} ; \mathrm{E}=\mathrm{S}$, Se [11]) nachgewiesen werden konnte, steht eine strukturelle Klärung für die VIII zugehörige Verbindungsklasse noch aus.

Einkristalle von VIII wurden aus $\mathrm{CH}_{2} \mathrm{Cl}_{2} / \mathrm{Hexan}(3 / 2)$ gewonnen und einer Röntgenstrukturanalyse unterzogen. Atomkoordinaten und ausgewählte Bindungsparameter sind in den Tabellen 2 und 3 aufgelistet. Bedingt durch die geringe Zahl von Reflexen und die statistische Verteilung der Co- und Rh-Atome von $1 / 1$ ist die Aussagekraft der Bindungsparameter etwas eingeschränkt. Eindeutig jedoch ist die Präsenz eines planaren $\mathrm{M}_{2} \mathrm{Se}_{2}$-Vierrings mit hierzu anti-ständigen $\mathrm{C}_{5} \mathrm{Me}_{5}$ - und CO-Liganden (Fig. 1). Eine analoge Stereochemie ist für $\mathrm{Cp}_{2}^{\star} \mathrm{Rh}_{2}(\mathrm{CO})_{2} \mathrm{Se}$ in Bezug auf den $\mathrm{M}_{2}$ Se-Dreiring gefunden worden [1]. Ein prinzipiell denkbares syn-Isomer konnte bei der Insertion eines Se-Atoms in die $\mathbf{R h}-\mathbf{R h}$-Bindung nicht isoliert

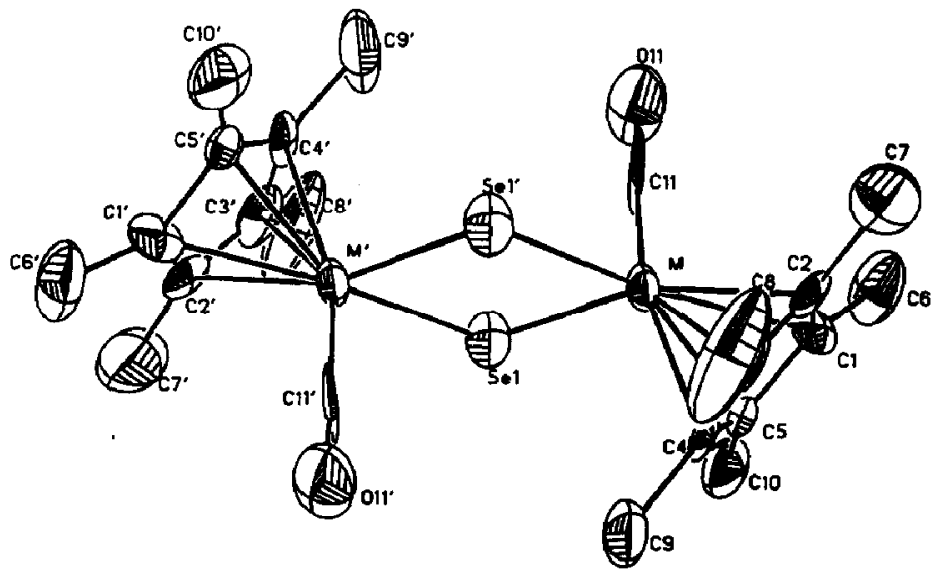

Fig. 1. Struktur von $\left(\mathrm{C}_{5} \mathrm{Me}_{5}\right)_{2} \mathrm{CoRh}(\mathrm{CO})_{2} \mathrm{Se}_{2}$ (VIII) im Kristall; die thermischen Ellipsoide entsprechen einer Wahrscheinlichkeit von $50 \%$. $M$ steht für $R$ h und $\mathrm{Co}(1 / 1$ statistisch verteilt). 
werden. Überraschend groß ist der Winkel an den Selenbrïcken $\left(101.3(1)^{\circ}\right)$. Strukturell charakterisierte $M_{2} E_{2}$-Vierringe, die weder eine $M-M-$ noch eine E-EWechselwirkung besitzen $(\mathrm{E}=\mathrm{Se}, \mathrm{Te})$ sind extrem selten. Sie zeichnen sich durch M-E-M-Winkel von etwas weniger als $90^{\circ}$ am Chalkogen E und E-E-Abstände über $3.5 \AA$ aus [12]. Dagegen liegen dieselben Winkel in $\left(\mathrm{PMe}_{3}\right)_{6} \mathrm{Co}_{2}(\mu \text {-Se })_{2}$ bei $103.6^{\circ}$ und der Se-Se-Abstand beträgt 2.893(2) A [13]. Da der Komplex entgegen den EAN-Regeln diamagnetisch ist, muß ein Spinaustausch über die Liganden erfolgen. Entsprechend verkürzt sind auch die M-Se-Abstände. In VIII erreichen zwar beide Metallatome Edelgaskonfiguration, dennoch scheint der Se-Se-Abstand von 3.055(3) $\AA$ auf intramolekulare Kontakte zwischen beiden Se-Atomen hinzudeuten. Ähnliche elektronische Verhältnisse wie in VIII liegen in $\mathrm{Cp}_{4} \mathrm{Ni}_{4} \mathrm{Se}_{2}\left(\mathrm{PPh}_{3}\right)_{2}$ vor, wo $d(\mathrm{Se}$...Se) 3.117(1) $\AA$ beträgt [14].

Eine weitergehende Analyse des Bindungssystems in VIII wird durch die Fehlordnung der beiden Metallatome leider verhindert.

\section{Experimenteller Teil}

Alle Arbeiten wurden unter Luftausschluß und unter Verwendung von trockenen $\mathrm{N}_{2}$-gesättigten Lösungsmitteln durchgeführt. Weitere Angaben zur Arbeitstechnik finden sich in Ref. [1]. Bei allen Versuchen wurde unter $\mathrm{N}_{2}$ "gealtertes" rotes, amorphes Selen verwendet. $\mathrm{Cp}_{2}^{\star} \mathrm{CoRh}(\mathrm{CO})_{2}$ (I) wurde durch Reaktion von $\mathrm{Cp}^{\star} \mathrm{Rh}(\mathrm{CO})_{2}$ mit $\mathrm{Cp}^{\star} \mathrm{Co}\left(\mathrm{C}_{2} \mathrm{H}_{4}\right)_{2}$ hergestellt (Petrolether 60/40; $110 \mathrm{~min}$ bei $55^{\circ} \mathrm{C}$; 84\% Ausbeute nach Chromatographie an $\mathrm{SiO}_{2}$ /Toluol) [15].

\section{Darstellung von $\mathrm{Cp}_{2}^{\star} \mathrm{CoRhS}_{8}(I I)$}

Die blaugrüne Lösung von $150 \mathrm{mg}(0.31 \mathrm{mmol}) \mathrm{Cp}_{2}^{\star} \mathrm{CoRh}(\mathrm{CO})_{2}$ und $79 \mathrm{mg}(0.31$ mmol) $S_{8}$ in $70 \mathrm{ml}$ THF wird 30 min bei Raumtemperatur gerührt, wobei sich die Farbe nach braun ändert. Nach Entfernen des Solvens wird der Rückstand in $10 \mathrm{ml}$ Toluol aufgenommen und an $\mathrm{Al}_{2} \mathrm{O}_{3}$ (Säule $8 \times 3 \mathrm{~cm}$ ) chromatographiert. Mit Toluol/ $\mathrm{Et}_{2} \mathrm{O}(15 / 1)$ eluiert man eine rotbraune Zone, die II in $60 \%$ Ausbeute enthält. Durch Umkristallisation aus Toluol/Pentan (1/1) erhält man schwarzbraune Kristalle.

Elementaranalyse: Gef.: C, 35.21; $\mathrm{H}, 4.43 . \mathrm{C}_{20} \mathrm{H}_{30} \mathrm{CoRhS}_{8}$ (688.8) ber.: C, 34.87; $\mathrm{H}, 4.39 \%$. Molmasse 656 (FD-MS aus Toluol; entspricht $[M-\mathrm{S}]^{+}$.

\section{Darstellung von $\mathrm{Cp}_{2}^{\star} \mathrm{CoRh}(\mathrm{CO})_{2} \mathrm{Se}_{2}$ (VIII)}

Die Lösung von $120 \mathrm{mg}(0.25 \mathrm{mmol}) \mathrm{I}$ und $59 \mathrm{mg}(0.75 \mathrm{mmol})$ Selen in $50 \mathrm{ml}$ THF wird 5 min bei $20^{\circ} \mathrm{C}$ gerührt. Der nach dem Abziehen des Lösungsmittels verbleibende dunkle Rückstand wird an $\mathrm{Al}_{2} \mathrm{O}_{3}$ (Säule $15 \times 3 \mathrm{~cm}$ ) chromatographiert. Mit Toluol eluiert man VIII als grünbraune Zone in $84 \%$ Ausbeute. Das so erhaltene Produkt wird aus $\mathrm{CH}_{2} \mathrm{Cl}_{2} / \mathrm{Hexan}(3 / 2)$ umkristallisiert.

Elementaranalyse: Gef.: C, 40.81; $\mathrm{H}, 4.89 ; \mathrm{C}_{22} \mathrm{H}_{30} \mathrm{O}_{2} \mathrm{CoRhSe}_{2}$ (646.2) ber.: C, 40.89; H, 4.68\%. Molmasse 648 (FD-MS aus Toluol; bzgl. ${ }^{80} \mathrm{Se}$ ).

\section{Darstellung von $\mathrm{CP}_{2}^{\star} \mathrm{CoRhSe_{5 }}$ (IX)}

Ein Gemisch aus $130 \mathrm{mg}(0.27 \mathrm{mmol}) \mathrm{I}, 168 \mathrm{mg}(2.13 \mathrm{mmol})$ Selen und $80 \mathrm{ml}$ THF wird $1 \mathrm{~h}$ bei Raumtemperatur gerührt. Zur Entfernung des überschüssigen Selens wird die braune Lösung über $\mathrm{Al}_{2} \mathrm{O}_{3}$ (Säule $7 \times 3 \mathrm{~cm}$ ) filtriert, wobei mit 50 
ml THF nachgewaschen wird. Nach Entfernen des Lösungsmittels nimmt man den Rückstand in $100 \mathrm{ml}$ Toluol auf und chromatographiert an $\mathrm{SiO}_{2}$ (Säule $18 \times 3 \mathrm{~cm}$ ). Mit Toluol eluiert man zunächst Spuren nicht identifizierbarer Verbindungen und dann mit Toluol/ $\mathrm{Et}_{2} \mathrm{O}(15 / 1)$ den gewünschten Komplex als braune Zone (Ausbeute 64\%). IX erhält man analysenrein durch Umkristallisieren aus Toluol/Pentan (1/1).

Elementaranalyse: Gef.: $\mathrm{C}, 28.50 ; \mathrm{H}, 3.64 . \mathrm{C}_{20} \mathrm{H}_{30} \mathrm{CoRhSe}_{5}$ (827.1) ber.: $\mathrm{C}$, 29.04; H, 3.66\%. Molmasse 832 (FD-MS aus Toluol; bzgl. ${ }^{80} \mathrm{Se}$ ).

\section{Röntgenographische Daten von Komplex VIII}

Grünbrauner Kristall $\left(0.38 \times 0.46 \times 0.53 \mathrm{~mm}^{3}\right)$, monoklin $C_{2 h}^{5}-P 2_{1} / n$; Zellkonstanten: $a$ 8.439(7), b 9.517(7), c 15.61(1) $\AA$, $\beta$ 104.64(7) ${ }^{\circ} ; V 1213.0 \AA^{3}, Z=2$; empirische Absorptionskorrektur (psi-scan Messung: 4 Reflexe $9.9<2 \theta<33.0^{\circ}$, Transmiss. Fakt. (min $/ \max ) 0.78 / 1.00), \mu=43.4 \mathrm{~cm}^{-1}, F_{000}=636, d$ (röntg) $=1.77$ $\mathrm{g} \mathrm{cm}^{-3}$; AED II der Fa. Siemens-Stoe [16]. Mo- $K_{\alpha}-$ Strahlung, Graphit-Monochromator, $\Omega$-scans, $h(0 / 11), k(0 / 12), l(-20 / 20)$, im vermessenen Bereich mögliche Reflexe $2180,1350 \geq 2 \sigma(I)$, unabhängige $1161(I \geq 2.5 \sigma(I))$. Die Struktur wurde gelöst mittels Patterson-, Fourier- und Differenzfouriersynthesen; alle Nichtwasserstoffatome wurden nach der Methode der kleinsten Quadrate anisotrop verfeinert, die Wasserstoffatome mit Hilfe des SHELXTL-Unterprogramms HFIX [17] fixiert; $R_{\text {anisotrop }}=0.068, \quad R_{\mathrm{w}}=0.057\left(R_{\mathrm{w}}=\left[\Sigma_{w}\left(\left|F_{\mathrm{o}}\right|-F_{\mathrm{c}} \mid\right)^{2} / \Sigma_{w} F_{\mathrm{o}}^{2}\right]^{1 / 2}\right) ; \quad$ Restelektronendichte $(\max / \mathrm{min}) 1.01 /-1.87 \mathrm{e} / \AA^{3}$, shift $/ \mathrm{esd}(\operatorname{mean} / \mathrm{max})=0.05 /-$ 0.48 , Goof $\left.=\left[\Sigma_{w}\left(\left|F_{\mathrm{o}}\right|-\left|F_{\mathrm{c}}\right|\right)^{2} / N O-N V\right)\right]^{1 / 2}=4.45$.

Alle Rechnungen basieren auf dem Programmsystem SHELXTL [17], die Atomformfaktoren wurden der Literatur entnommen [18].

\section{Literatur}

1 H. Brunner, N. Janietz, W. Meier, J. Wachter, E. Herdtweck, W.A. Herrmann, O. Serhadli und M.L. Ziegler, J. Organomet. Chem., 347 (1988) 237.

2 J. Wachter, J. Coord. Chem. B, 15 (1987) 219.

3 K. Endrich, E. Guggolz, O. Serhadli, M.L. Ziegler und R.P. Korswagen, J. Organomet. Chem., 349 (1988) 323.

4 Wie auch für $\mathrm{CP}_{2}^{\star} \mathrm{Rh}_{2} \mathrm{~S}_{8}$ [5] wird für II im FD-Massenspektrum nur der um ein S-Atom verringerte Molekülpeak beobachtet.

5 H. Brunner, N. Janietz, W. Meier, B. Nuber, J. Wachter und M.L. Ziegler, Angew. Chem., 100 (1988) 717; Angew. Chem. Int. Ed. Engl., 27 (1988) 708.

6 H. Brunner, N. Janietz, W. Meier, G. Sergeson, J. Wachter, T. Zahn und M.L. Ziegler, Angew. Chem., 97 (1986) 1056; Angew. Chem. Int. Ed. Engl., 24 (1985) 1060.

7 W.A. Herrmann, C. Bauer und J. Weichmann, Chem. Ber., 117 (1984) 1271; W.A. Herrmann und J. Weichmann, Organomet. Synth., 3 (1986) 287.

8 Bei dieser Betrachtungsweise sind die Brückenliganden formal als ungeladen anzusehen.

9 M. Herberhold und B. Schmidkonz, J. Organomet. Chem., 358 (1988) 301.

10 H. Brunner, W. Meier, B. Nuber, J. Wachter und M.L. Ziegler, Angew. Chem., 98 (1986) 907; Angew. Chem. Int. Ed. Engl., 25 (1986) 908.

11 M. Herberhold, D. Reiner, B. Zimmer-Gasser und U. Schubert, Z. Naturforsch. B, 35 (1980) 1281.

12 F. Bottomley, T.-T. Chin, G.O. Egharevba, L.M. Kane, D.A. Pataki und P.S. White, Organometallics, 7 (1988) 1214; G. Erker, T. Mühlenbernd, R. Nolte, J.L. Petersen, G. Tainturier und B. Gautheron, J. Organomet. Chem., 314 (1986) C21.

13 H.F. Klein, M. Gaß, U. Koch, B. Eisenmann und H. Schäfer, Z. Naturforsch, B, 43 (1988) 830.

14 D. Fenske, A. Hollnagel und K. Merzweiler, Angew. Chem., 100 (1988) 978; Angew. Chem. Int. Ed. Engl., 27 (1988) 965. 
15 M. Green, D.R. Hankey, J.A.K. Howard, P. Louca und F.G.A. Stone, J. Chem. Soc., Chem. Commun., (1983) 757.

16 STRUCSY, Structure System Program Package, Fa. Stoe, Darmstadt, FRG, 1984.

17 G.M. Sheldrick, SHELXTL-Programm, Universität Göttingen, FRG, 1983.

18 International Tables for X-Ray Crystallography, Vol. IV, The Kynoch Press, Birmingham, 1974. 